\title{
technische universität dortmund
}

Hartmut Hirsch-Kreinsen

\section{MULTINATIONALE UNTERNEHMEN}

Soziologisches Arbeitspapier Nr. 23/2008

Herausgeber

Prof. Dr. H. Hirsch-Kreinsen Prof. Dr. J. Weyer 


\section{Multinationale Unternehmen}

Hartmut Hirsch-Kreinsen

Arbeitspapier Nr. 23 (September 2008)

ISSN 1612-5355 


\section{Herausgeber:}

Prof. Dr. Hartmut Hirsch-Kreinsen

Lehrstuhl Wirtschafts- und Industriesoziologie

is@wiso.tu-dortmund.de

www.wiso.tu-dortmund.de/IS
Prof. Dr. J ohannes Weyer

Fachgebiet Techniksoziologie johannes.weyer@tu-dortmund.de www.wiso.tu-dortmund.de/TS

Wirtschafts- und Sozialwissenschaftliche Fakultät

Technische Universität Dortmund

D-44221 Dortmund

\section{Ansprechpartnerin:}

Dipl.-Päd. Martina Höffmann, e-mail: martina.hoeffmann@tu-dortmund.de

Die Soziologischen Arbeitspapiere erscheinen in loser Folge. Mit innen werden Aufsätze (oft als Preprint), sowie Projektberichte und Vorträge publiziert. Die Arbeitspapiere sind daher nicht unbedingt endgültig abgeschlossene wissenschaftliche Beiträge. Sie unterliegen jedoch in jedem Fall einem internen Verfahren der Qualitätskontrolle. Die Reihe hat das Ziel, der Fachöffentlichkeit soziologische Arbeiten aus der Wirtschafts- und Sozialwissenschaftlichen Fakultät der Technischen Universität Dortmund vorzustellen. Anregungen und kritische Kommentare sind nicht nur willkommen, sondern ausdrücklich erwünscht. 


\section{Multinationale Unternehmen ${ }^{1}$}

Hartmut Hirsch-Kreinsen

\section{Einleitung}

Gegenstand des folgenden Kapitels sind multinationale Unternehmen. In der sozialwissenschaftlichen Globalisierungsdebatte werden diese Unternehmen als die treibenden Akteure der fortschreitenden Globalisierung angesehen; sie seien die zentralen Akteure in einer weltweiten „interlinked economy“ (z.B. Ohmae 1990; Dunning 1993). Es wird vom Aufkommen einer globalisierten Produktion gesprochen, deren Hauptkennzeichen internationale Direktinvestitionen, die technologische Zusammenarbeit zwischen internationalen Konzernen und die Entstehung von globalen Netzwerken und Wertschöpfungsketten sind (Altvater/Mahnkopf 1997: 23). Einer Minimaldefinition zu Folge wird von einem multinationalen Unternehmen (MNU) dann gesprochen, wenn es in zwei oder mehr unterschiedlichen nationalen Einflussbereichen organisatorisch präsent ist. Der Begriff multinationales Unternehmen ist allgemein gebräuchlich und es werden damit nicht eine bestimmte Unternehmensstrategie und eine Organisationsstruktur angesprochen. Zudem ist es grundsätzlich unerheblich, ob es sich um ein großes oder ein kleines Unternehmen handelt (Morgan 2005: 555).

Die sozialwissenschaftliche Forschung über multinationale Unternehmen steht spätestens seit der Mitte der 1990er Jahre im Kontext der generellen Globalisierungsdebatte und lässt sich disziplinär nur schwer eindeutig zuordnen. Es verschränken sich managementwissenschaftliche, wirtschaftsgeographische und regionalsoziologische sowie organisations- und industriesoziologische Analysen. Hervorzuheben ist hier zunächst die international vergleichende Managementforschung, die sich zumeist in einer praxeologischen Gestaltungsperspektive mit den Strukturen und Strategien multinationaler Unternehmen befasst (z.B. Porter 1986; Bartlett/Goshal 1989; Ghemewat 2007; zusammenfassend: Welge/Holtbrügge 2003).

1 Erscheint demnächst in: Böhle, Fritz/Voß, Günther, G./Wachtler, Günther (Hrsg): Handbuch Arbeitssoziologie, Wiesbaden: VS Verlag (2009). 
Oftmals eng verbunden damit sind Studien wirtschaftsgeographischer und regionalsoziologischer Provenienz, die sich mit der Bedeutung regionaler Unternehmensstandorte und Cluster für internationale Unternehmen befassen (z.B. Scott 1996; Porter 1998; Schamp 2000).

Zweifellos haben diese beiden eher ökonomischen Forschungsrichtungen die soziologische Unternehmensforschung stark beeinflusst und befruchtet, insofern als sie sehr hilfreiche Vorschläge zur Charakterisierung spezifischer Typen multinationaler Unternehmen vorgelegt hat (Pries 2008: 182). Daran orientiert, befasst sich die soziologische Analyse mit den verschiedenen Erscheinungsformen multinationaler Unternehmen und ihren sozialen Voraussetzungen und Konsequenzen: $\mathrm{Zu}$ nennen sind zum einen Studien, die sich mit den Internationalisierungsstrategien von Großunternehmen aus den Kernbranchen wie der Automobilindustrie (z.B. Pries 1999; Boyer/Freyssenet 2003) und der Telekommunikationsbranche (z.B. Dörrenbächer 1999) der deutschen Industrie befassen oder den Prozess des Organisationswandels in einer vergleichenden Perspektive aufgreifen (z.B. Beyer 2001). Thematisiert werden zum zweiten Fragen nach den Konsequenzen der institutionellen Einbettung internationaler Unternehmen für ihre Struktur- und Strategiemuster und der Bedeutung regionaler Bindungen für solche Unternehmen (z.B. Ruigrok/Tulder 1995; Hirsch-Kreinsen/Wannöffel 2003; Wagner/Mense-Petermann 2006). Zum dritten wird Fragen, die sich auf Management- und Koordinationsproblemen bzw. den Politik- und Machtprozessen in internationalen Unternehmen richten, nachgegangen (z.B. von Behr/Hirsch-Kreinsen 1998; Dörrenbächer 2003; Geppert/Mayer 2006). Zum vierten werden die Konsequenzen der Internationalisierungsstrategien für das System der industriellen Beziehungen untersucht (z.B. Schmierl 1998; Müller et al. 2006). Und schließlich wird gefragt, inwieweit von einer Internationalisierung des Managements von Unternehmen gesprochen werden kann (z.B. Hartmann 1999; Caroll/Fennema 2002). Diese vielfältigen und breiten Forschungsergebnisse sollen im Folgenden in Hinblick auf zentrale Ergebnisse resümiert werden. 


\section{Strategien und Strukturen von MNU}

\subsection{Historische Entwicklung}

Wie die Forschung immer wieder betont, ist das Aufkommen multinationaler Wirtschaftsorganisationen keineswegs neueren Datums (z.B. Cameron 1992; Held et al. 2005; Morgan 2005; Abele et al. 2006). Datiert wird dies verschiedentlich auf das späte 17. und frühe 18. Jahrhundert und die damaligen Export- und Importaktivitäten großer internationaler Handelsunternehmen, typisch hierfür die East India Trading Company. Stabile multinationale Unternehmensstrukturen entwickelten sich allerdings erst mit Auslandsinvestitionen und der damit verbundenen Gründung von ausländischen Tochtergesellschaften und Kapitalbeteiligungen an ausländischen Unternehmen. Allerdings verlief dieser Prozess in unterschiedlichen Mustern:

Die dominante Form der Auslandsinvestitionen in der ersten Hälfte des 19. Jahrhunderts waren sog. Portfolioinvestitionen; vermittelt durch Investitionsbanken (insbesondere der Londoner City) investierten Unternehmen in Kapitalbeteiligungen etwa an ausländischen Infrastrukturunternehmen wie Eisenbahngesellschaften. Parallel entstanden dazu Formen direkter Auslandsinvestitionen, die sich primär auf die im Kontext des kolonialen Zeitalters besonders ausgeprägte Rohstoffgewinnung und -erzeugung richteten. Typische MNUs dieser Zeit waren Europäische und USamerikanische Großunternehmen wie Royal Durch Shell, British Petroleum oder Dunlop. Es handelte sich um politisch und ökonomisch sehr einflussreiche Großunternehmen, die weitgehend unabhängig von ihrer nationalen Herkunft ihre strategischen Ziele im Kontext des kolonialen Zeitalters verfolgten. Begleitet waren diese Aktivitäten von der internationalen Ausweitung von Finanzunternehmen, wodurch der Zugang zu dem erforderlichen Investitionskapital erleichtert wurde. Multinationale Produktionsunternehmen waren hingegen im 19. Jahrhundert, sieht man von einigen Europäischen Ausnahmen wie etwa dem elektrotechnischen Unternehmen Siemens und Halske ab, eher die Ausnahme. Diese Entwicklung wird in der Zwischenkriegszeit auf Grund der Weltwirtschaftskrise und der protektionistischen Politik vieler Staaten weitgehend abgebrochen.

In der ersten Zeit nach dem Zweiten Weltkrieg wurden die ökonomischen Beziehungen zwischen den verschiedenen Ländern vornehmlich vom Handel geprägt. 
Unterstützt von einer ständigen politischen Liberalisierung der Wirtschaftsbeziehungen vervierfachte der Welthandel zwischen dem Ende der 40er und dem Ende der 60er Jahre sein Volumen, wobei 1957 der Welthandel mit Investitionsgütern historisch zum ersten Mal den mit Produkten des primären Wirtschaftssektors überstieg (Kennedy 1991: 616). Vorangetrieben wurde diese Entwicklung von den Exportaktivitäten vieler Unternehmen aus den Industrieländern. Neben den Handelsströmen fanden sich in begrenztem Rahmen auch internationale Direktinvestitionen in Form von Unternehmensaufkäufen, Beteiligungen an Unternehmen und die Neugründung von Produktionsstätten. In den 50er und 60er Jahren wurde diese Strategie vor allem von US-Konzernen verfolgt, neben General Motors und Ford ist hier als Beispiel General Electric zu nennen. Sie brachten Ende 1966 mehr als 60\% aller internationalen Direktinvestitionen auf und konzentrierten sich auf die damaligen Wachstumsmärkte Westeuropa. Multinationale Unternehmen westeuropäischer Herkunft gab es lediglich aus Ländern mit einer schon lange zurückreichenden internationalen Orientierung ihrer ökonomischen Aktivitäten wie den Niederlanden oder Großbritannien; zu nennen sind hier etwa Unternehmen wie Royal Dutch/Shell, British Petroleum, Philips und Unilever, die teilweise schon seit dem Beginn des Jahrhunderts international aktiv waren (Ruigrok/van Tulder 1995: $128 \mathrm{ff}$.$) . Westdeutsche Unternehmen spielten demgegenüber nur eine$ nachgeordnete Rolle und die westdeutschen Direktinvestitionen beliefen sich 1966 nur auf 3\% der weltweiten Direktinvestitionen (Busch 1974: 117).

Spätestens seit dem Beginn der 1980er Jahre intensivierten sich die internationalen ökonomischen Verflechtungen. Wirksam war hier ein ganzes Bündel sehr unterschiedlicher Faktoren: Erstens drängte die zunehmende Sättigung der Nachfrage auf den nationalen Absatzmärkten vieler westlicher Länder die Unternehmen verstärkt international aktiv zu werden. Zweitens wuchs auf den Märkten für einfache, in arbeitsintensiven Produktionsprozessen herstellbare Produkte die Konkurrenz von Unternehmen aus bislang kaum auf dem Weltmarkt in Erscheinung getretenen neuindustrialisierten Ländern heran. Dies zwang viele Unternehmen dazu, systematisch internationale Kosten- und Wachstumsdifferenzen auszunutzen, um ihre jeweilige Konkurrenzposition zu verbessern. Drittens erleichterten politische Gründe wie die Öffnung von national abgeschotteten Märkten 
und der Abbau von Zollgrenzen internationale Unternehmensaktivitäten. Viertens verringerte ein deutlicher Rückgang von Transport- und Kommunikationskosten Barrieren für die Internationalisierung von Unternehmen.

Manche Autoren sprechen davon, dass diese Faktoren zu einer "dammbruchartigen“ Entwicklung führten und viele Industriebranchen in kurzer Zeit radikal umgebaut worden seien (Abele et al. 2006: 10). Ein sicherlich dramatisches Beispiel hierfür ist die Erosion der Textil- und Bekleidungsindustrie in den alt industrialisierten Ländern und ihre schnelle Verlagerung in Entwicklungs- und Schwellenländer seit Beginn der 1970er Jahre (Fröbel et al. 1977). Seit Mitte der 80er Jahre lässt sich ein stark ansteigendes Volumen internationaler Direktinvestitionen aus den westlichen Industrieländern beobachten. Dieser Anstieg schwächte sich zwar zu Beginn der 2000er J ahre krisenbedingt ab, doch nimmt er einige Jahre später wieder Fahrt auf. Vorliegenden internationalen Daten zu Folge (alle folgenden Daten aus: UNCTD 2007: Table 2) stieg das Gesamtvolumen im Ausland produzierter Güter und Dienstleistungen deutlich schneller an als in den Jahren zuvor. In Hinblick auf die sektorale Verteilung von Auslandsinvestitionen zeigt sich dabei ein massiver Anstieg der Anteile des Dienstleistungssektors, insbesondere von Infrastrukturunternehmen; Unternehmen aus diesem Sektor bestreiten 2005 fast zwei Drittel aller ausländischen Direktinvestitionen, im Vergleich dazu waren es 1990 nur rd. 49\%. Der Anteil des industriellen Sektors an den Auslandsinvestitionen belief sich 2005 auf etwa 30\%, während es 1990 noch rd. 41\% waren. Im Jahr 2006 sind die Umsätze, Produktionswerte und Exporte der rund 78.000 MNUs und ihrer ca. 780.000 ausländischen Niederlassungen um bis zu 20\% angestiegen. Der Produktionswert internationaler Unternehmen beläuft sich in dem genannten Jahr auf ca. 10\% des weltweiten BSP und MNUs bestreiten ungefähr ein Drittel des Welthandels. Zwar liegen die geografischen Schwerpunkte der von multinationalen Unternehmen getätigten Auslandsinvestitionen nach wie vor in den ökonomischen Zentren, Europa, Nordamerika und Südostasien, jedoch ist zugleich eine Verschiebung der Auslandsaktivitäten in südliche Gegenden des Globus zu beobachten. So beherbergt China die meisten Auslandsniederlassungen multinationaler Unternehmen. Zudem expandieren zunehmend auch MNUs aus Niedriglohnländern auf dem Weltmarkt. Es 
handelt sich dabei beispielsweise um Unternehmen aus der Bau- und Stahlindustrie sowie der IT-Industrie (Abele et al. 2006: 157).

\subsection{Strategievarianten}

Die sozialwissenschaftliche Unternehmensforschung präzisiert die Annahme über die wachsende Bedeutung multinationaler Unternehmen und ihrer Strukturen und Strategien wie folgt: Es werden verschiedene typische Strategievarianten multinationaler Unternehmen herausgearbeitet, wobei betont wird, dass die Strategievarianten realiter selten in ihrer idealtypischen Form anzutreffen seien. Vielmehr variieren sie nach Branche, Produkten und Herkunftsland (z.B. Porter 1986; Bartlett/Goshal 1989). Zugleich wird aber mit diesen Varianten eine Entwicklungshypothese verbunden, der zu Folge diese verschiedene und aufeinander folgende Phasen der Entwicklung multinationaler Unternehmen bezeichnen (zusammenfassend Welge/Holtbrügge 2003: 128ff.).

Als erste Variante werden exportorientierte Unternehmen bzw. internationale Unternehmen genannt, die in hohem Maße von einem national lokalisierten Standort aus operieren. Allenfalls sind diese Unternehmen durch Service- und Verkaufsniederlassungen im Ausland vertreten. Diese Strategievariante spielt fraglos, so etwa im deutschen Maschinenbau, bis heute eine große Rolle, doch wird davon ausgegangen, dass er kaum mehr in der Ausschließlichkeit wie früher anzutreffen ist.

Eine zweite, früher häufig anzutreffende Strategievariante wird als klassisch multinationa/ begriffen; Unternehmen mit dieser Strategie sind von einem losen, über Jahrzehnte gewachsenen Konglomerat von Zentrale und starken Auslandsgesellschaften gekennzeichnet. Besonderes Merkmal dieser Strategievariante ist eine Anpassung der Auslandsniederlassungen an die Bedingungen des jeweiligen Gastlandes, was in der Regel durch spezifische Organisationsstrukturen und Produktstrategien erreicht werden soll. Daher ist eine konzernweite Integration der einzelnen Auslandsgesellschaften nur begrenzt möglich und wird in diesem Fall in der Regel auch nicht angestrebt. Solche Strategien fanden sich vornehmlich in Europa bei Konzernen der elektrotechnischen Industrie und der Nahrungsmittelindustrie, aber auch bei amerikanischen Automobilkonzernen wie General Motors und Ford. 
Ausgegangen wird nun von einem Wandlungsprozess dieser Strategie. Zum einen findet sich danach die Variante eines globalen Unternehmens, das auf weltweit homogene Marktsegmente abzielt und Integration auf dem Weg einer tendenziell weltweiten Standardisierung von Produktion und Produkten und einer möglichst weitgehenden Zentralisierung von Entscheidungen und Funktionen anstrebt. Konkurrenzvorteile sollen hier auf der Basis der "economies of scale", einer deutlichen Verringerung der Fertigungstiefe in den einzelnen Produktionsstätten und einer gezielten Nutzung regionaler und länderspezifischer Kostenvorteile durch den Aufbau globaler Zulieferbeziehungen erreicht werden. Bezeichnet wird damit ein Typus von Unternehmen, der beispielsweise seit dem Ende der 1970er Jahre in der international ausgerichteten japanischen Photoindustrie und Unterhaltungselektronik anzutreffen war.

Auf der anderen Seite wird die Variante eines transnationalen Unternehmens hervorgehoben. Als zentrales Merkmal dieses Unternehmenstypus gilt die Ausdifferenzierung bislang integrierter, auf einzelne Länder konzentrierter Unternehmen und die Lokalisierung von Produktionsstätten in den wichtigsten Weltregionen und Segmenten des Weltmarktes. Zum einen sollen dadurch Kostendifferenzen zwischen verschiedenen Ländern und Regionen für eine global orientierte Produktivitätssteigerung genutzt werden. Zum zweiten können damit die risikoreichen Währungsturbulenzen und weitere unkalkulierbare Barrieren des Welthandels umgangen und durch die räumliche und soziale Nähe der Produktion zu den Käufern in den verschiedenen Segmenten des Weltmarktes bisherige Absatzchancen gesichert und neue erschlossen werden. Zum dritten wird mit dieser Form der Internationalisierung die Voraussetzung dafür geschaffen, Innovationsprozesse im internationalen Maßstab zu reorganisieren und zu rationalisieren, um sie nachhaltig zu beschleunigen; einschlägiges Stichwort ist die kontinuierliche Verkürzung der "time to market", d.h. Produktinnovationen schneller als Konkurrenzunternehmen auf den Markt zu bringen.

Diese Zielsetzungen erfordern, so weitere Merkmale dieser Strategievariante, eine tendenziell weltweit verteilte, zugleich elastische wie aber auch steuerbare Unternehmens- und Produktionsstruktur. Organisatorisch verbindet sich daher mit der transnationalen Strategie ein Netzwerk ausdifferenzierter Produktions- und 
Wertschöpfungseinheiten, deren Funktionen durch neuartige Formen informationstechnisch gestützter Koordinations- und Steuerungsmechanismen aufeinander abgestimmt und integriert werden. Als wesentliches Merkmal gilt daher auch die Ausweitung und Intensivierung regionaler und lokaler Aktivitäten und zugleich ihre umfassende Abstimmung und Integration auf der Ebene einer international agierenden Produktions- und Wertschöpfungskette.

Als prominentes Beispiel für den Typus eines transnationalen Unternehmens gilt bis heute das schwedisch-schweizerische elektrotechnische Unternehmen Asea Brown Boveri (ABB AG) (v. Behr/Hirsch-Kreinsen 1998; Bélanger et al. 1999; Morgan 2005). In der zweiten Hälfte der 1990er Jahre galt ABB als Modell für einen überaus erfolgreichen "Global Player". Ständige Produktionsverlagerung, Dezentralisierung, kontinuierliche Rationalisierung, insgesamt eine äußerst hohe Organisationsdynamik waren seine Besonderheiten. Das zentrale Merkmal dieses Unternehmens war seine spezielle Organisationsstruktur, eine Matrix, durch die offensichtlich ein permanenter Wandel und schnelle Anpassung des Unternehmens an neue Bedingungen möglich war.

In Anlehnung an die Ergebnisse aus der international vergleichenden Managementforschung (Welge/Holtbrügge 2003: 129; Abele et al. 2006: 170) kann das skizzierte Spektrum der verschiedenen Strategievarianten mit den beiden Dimensionen des organisatorischen und unternehmensstrategischen Integrationsgrad einerseits und dem Grad der regionalen Differenzierung von Unternehmen auf dem Weltmarkt andererseits in der folgenden schematischen Abbildung (Abb. 1) zusammengefasst werden. Wie schon angesprochen, wird damit zugleich eine Entwicklungshypothese verbunden. Ausgegangen wird davon, dass die verschiedenen Strategietypen aufeinander folgende Phasen eines Entwicklungsprozesses internationaler Unternehmen bezeichnen. 


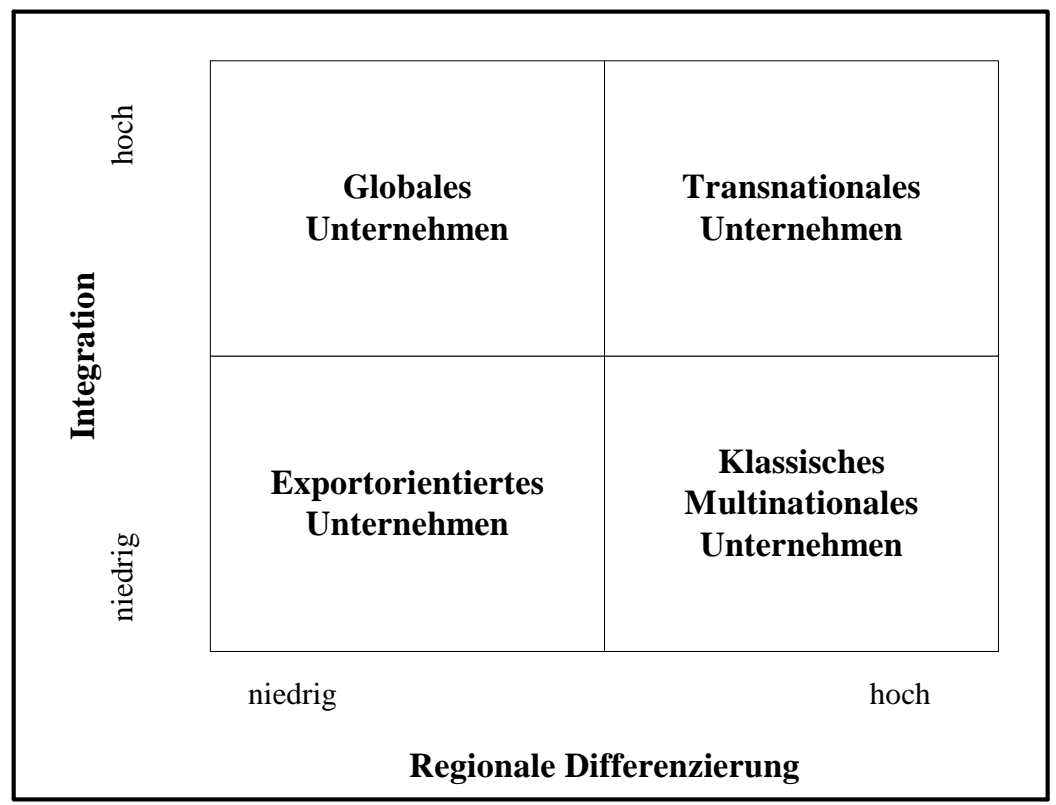

Abb. 1: Strategievarianten multinationaler Unternehmen

\subsection{Differenzierungen}

Freilich legen die vorliegenden Forschungsergebnisse eine Differenzierung der skizzierten Entwicklungshypothese multinationaler Unternehmen in verschiedener Hinsicht nahe:

\section{a) Divergente Unternehmensstrukturen}

Eine Reihe neuerer Untersuchungsergebnisse bestätigen ein abnehmendes Gewicht exportorientierter Unternehmen, die ausschließlich von nationalen Standorten aus operieren, und eine anwachsende Bedeutung ausdifferenzierter, netzwerkförmiger Unternehmensstrukturen insbesondere bei größeren international agierenden Unternehmen (Kinkel/Lay 2000; Beyer 2001; Hirsch-Kreinsen/Wannöffel 2003). Zugleich aber wird deutlich, dass sie sich je nach Branche, Unternehmensgröße und Stellung des Unternehmens in der Wertschöpfungskette organisatorisch unterscheiden. Eine Konvergenz der Strategien und Organisationsstrukturen multinationaler Unternehmen ist allen vorliegenden Befunden zu Folge nicht erkennbar. Verschiedentlich wird sogar von einer Tendenz hin zu einer zunehmenden Varietät der Organisationsstrukturen multinationaler Unternehmen ausgegangen (z.B. Beyer 2001, 2006; Ghemawat 2007). Divergenzen betreffen zum einen den Grad ihrer internationalen Dezentralisierung und die je konkrete Form der realisierten 
internationalen Unternehmensnetzwerke. Zum anderen gewinnen allen vorliegenden Forschungsergebnissen zu Folge an bestimmten Standorten unter spezifischen Bedingungen konzentrierte Unternehmensstandorte wieder an Bedeutung. Solche zentralisierten "Weltfabriken" finden sich neuerdings in Hightech Industrien wie der Halbleiterfertigung, der Computerherstellung und der Montage von Großflugzeugen. Damit sollen vor allem Verbund- und Skaleneffekte und die effektive Nutzung von hoch spezialisierten Personal und Know-how sicher gestellt werden (Abele et al. 2006: 169ff.).

Zudem sind große Differenzen in Hinblick auf die Reichweite der internationalen Ausdehnung zu beobachten. Während Unternehmen beispielsweise aus der Automobil- und Chemieindustrie versuchen, ihre Produktionsstätten in allen wichtigen Regionen des Weltmarktes zu lokalisieren, konzentrieren Unternehmen aus anderen Branchen und viele mittlere und kleinere Unternehmen ihre Aktivitäten nur auf bestimmte Regionen. Ein Grund hierfür sind sicherlich unterschiedliche Nachfrageund Absatzstrukturen wie aber auch unterschiedliche Ressourcen, die mit der Unternehmensgröße erheblich differieren und insbesondere kleineren Unternehmen geringere Handlungsspielräume eröffnen. Diese schlagen sich naturgemäß auch in nur begrenzten internationalen Aktivitäten von kleineren und mittleren Unternehmen nieder. Dies lässt sich mit Daten über Produktionsverlagerungen ins Ausland belegen: So verlagerten im Zeitraum zwischen 2004 und 2006 im Verarbeitenden Gewerbe Deutschlands nur rd. 10\% der kleinen Betriebe mit weniger als 100 Beschäftigten Teile der Produktion ins Ausland, während dies für mehr als die Hälfte der Betriebe mit mehr als 500 Beschäftigten zutraf (Kinkel/Maloca 2008).

\section{b) Koordinationsprobleme und Rückverlagerung}

Vorliegende Daten zeigen zudem, dass internationale Aktivitäten von Unternehmen keinen unumkehrbaren, nur in Richtung eines kontinuierlich sich intensivierenden und geographisch sich ausweitenden Prozess darstellen. Konkret lässt sich das an Rückverlagerungen von vormals ausgelagerten Produktionseinheiten festmachen: So kommt im deutschen Verarbeitenden Gewerbe zwischen 2004 und 2006 etwa auf jeden sechsten Verlagerer ein rückverlagernder Betrieb. Es handelt sich dabei um Unternehmen, die ihr Auslandsengagement einschränken und Produktionskapazitäten an einen inländischen Standort zurück holen (Schulte 2002). Als Anlass hierfür 
werden insbesondere Korrekturen von Fehleinschätzungen des Unternehmensmanagements über die ökonomischen Vorteile von Internationalisierungsaktivitäten gesehen (Kinkel/Maloca 2008: 4ff.). Solche Fehleinschätzungen resultieren oft aus kurzfristig motivierten und ausschließlich am Ziel der Kostenminimierung orientierten Managemententscheidungen, die die Komplexität und Folgekosten von Internationalisierungsaktivitäten vernachlässigen (z.B. Kinkel 2004). Insbesondere sind sie Ergebnis von Koordinations- und Managementproblemen multinationaler Unternehmen, die je nach konkreter Struktur, Differenziertheit und geographischer Ausdehnung eines Unternehmens unterschiedlich auftreten. Sie haben oft den Charakter unerwarteter Folgen der Internationalisierungsentscheidungen des Managements und sie können nachhaltige kontraproduktive Effekte für die Unternehmen entfalten. Diese Koordinationsprobleme umfassen beispielsweise: Schwierigkeiten beim länderübergreifenden Transport und der Logistik, Abstimmungs- und Integrationsbarrieren zwischen den Standorten aus verschiedenen Ländern auf Grund unterschiedlicher Organisationsstrukturen, Arbeitsmethoden, Managementpraktiken, generell Unternehmenstraditionen, Widerstände und Beharrungskräfte gegen die Internationalisierung und Integration verschiedener Standorte in einen länderübergreifenden Unternehmensverbund und schließlich die Schwierigkeit die unterschiedlichen politischen und gesellschaftlichen Bedingungen der einzelnen Standorte wie etwa die Regelungen der länderspezifischen Systeme der industriellen Beziehungen auf Unternehmensebene miteinander kompatibel zu machen.

\section{c) Standortbindungen}

Der sozialwissenschaftlichen Unternehmensforschung zu Folge lassen sich die turbulenten und häufig unkalkulierbaren Anforderungen des Weltmarktes, die auf eine Steigerung der Flexibilität, Verbreiterung der Produktpalette und Beschleunigung der Innovationen drängen, nur dann bewältigen, wenn die internationalen Unternehmen über relativ stabile lokale Bindungen verfügen. In diesem Sinn werden lokale Bindungen geradezu als Voraussetzung einer erfolgreichen Globalisierung angesehen (z.B. Scott 1996; Porter 1998; Ghemawat 2007). Die Bedeutung lokaler Bindungen ergibt sich daraus, dass über eingespielte und enge Beziehungen zu anderen in der gleichen Region angesiedelten Unternehmen und Institutionen ein 
relativ problemloser Zugriff auf zusätzliche, für Internationalisierungsaktivitäten benötigte Ressourcen wie etwa Arbeitskräfte mit speziellen Qualifikationen, Beratung und Wissen wie aber auch finanzieller Unterstützung möglich ist. Häufig sind diese Standortfaktoren Teil eines umfassenderen regionalen Strukturzusammenhangs mit weiteren Industriebranchen, Zulieferbetrieben und wichtigen Kunden, in dem die Unternehmen eingebettet sind. Solchermaßen verstandene territoriale Bindungen realisieren sich in der Regel innerhalb geographisch mehr oder weniger eingrenzbarer Regionen, die unterhalb oder oftmals auch quer zu nationalstaatlichen Grenzen angesiedelt sind. Mit unterschiedlichen Konnotationen wird hier von Clustern, industriellen Distrikten und regionalen Agglomerationen gesprochen.

Die internationale Ausrichtung der Unternehmen wird daher auch als Wechselspiel zwischen ihrer fortschreitenden internationalen Ausdehnung einerseits und dem Wandel und Erhalt lokaler Bindungen andererseits aufgefasst. Die Forschungsergebnisse verdeutlichen, dass die "Home Base" der internationalen Unternehmen eine wesentliche Voraussetzung für erfolgreiche Internationalisierungsaktivitäten darstellt und dass gerade auch internationale Großunternehmen bis heute vergleichsweise stark lokal bzw. national eingebunden sind. Empirisch festmachen lässt sich dies beispielsweise an der vielfach beobachtbaren Dominanz von Managern aus dem Herkunftsland besonders im Topmanagement der Unternehmen (Hartmann 1999) und dem Erhalt nationalspezifisch eingefahrener Praktiken der Arbeitspolitik und der industriellen Beziehungen.

\section{d) Überbetriebliche Wertschöpfungsketten}

Die Forschungsergebnisse machen zugleich deutlich, dass internationale Unternehmen auch an ihren Auslandsstandorten den Bezug zu den dort herrschenden regionalen und nationalen Bedingungen herstellen müssen, wenn sie kontraproduktive Nebenfolgen ihres Auslandsengagements vermeiden und wirkliche Konkurrenzvorteile erzielen wollen. Damit wird einmal mehr die große Bedeutung des oben beschriebenen transnationalen Unternehmens (Abschn. 2.2) mit einer ausdifferenzierten Wertschöpfungskette, die sich auf die Nutzung heterogener Standortbedingungen im internationalen Maßstab richtet, unterstrichen. Die Relevanz heterogener Standortbedingungen für den ökonomischen Erfolg multinationaler Unternehmen ist auch der Grund dafür, dass einzelne Unternehmen mehr und mehr 
in internationale überbetriebliche Wertschöpfungsketten eingebunden sind. Es wird von einer zunehmenden internationalen Fragmentierung von Produktionsprozessen gesprochen, deren Teilfunktionen aufeinander abgestimmt von verschiedenen Unternehmen in unterschiedlichen Ländern ausgeführt werden (z.B. OECD 2008; Sturgeon et al. 2008). Diese Entwicklung ist Folge der inzwischen weit verbreiteten Strategie des "Outsourcing" und "Offshoring" von Unternehmensfunktionen auf internationaler Ebene. Diese Begriffe bezeichnen die Auslagerung bestimmter Unternehmensfunktionen an ausländischen Unternehmen und umgekehrt die Beschaffung von Zwischenprodukten oder Dienstleistungen von dafür spezialisierten ausländischen Anbietern. Die Austauschprozesse innerhalb dieser fragmentierten Wertschöpfungsketten sind einerseits marktförmig geregelt, andererseits aber ist der Übergang zu engeren Netzwerkstrukturen mit ungleichen Machtbeziehungen zwischen Endproduzenten und Zulieferern, aber auch wechselseitigen technologischen und ökonomischen Abhängigkeiten offenbar fließend. Als technologische Voraussetzung für die relative Formalisierung und die Funktionsfähigkeit solcher Wertschöpfungsbeziehungen wird die Standardisierung bzw. Modularisierung der Zwischenprodukten angesehen, wodurch aufwendige und es ante nur schwer zu kalkulierende Abstimmungs- und Kooperationsprozesse unnötig werden (Faust et al. 2004).

Eine Folge dieser Entwicklung ist, dass der Welthandel zunehmend vom Handel mit Zwischenprodukten geprägt ist; vorliegenden Zahlen zu Folge umfasste weltweit im Jahr 2003 der Handel mit solchen Produkten rd. 54\% des gesamten Welthandels industriell gefertigter Güter. Betroffen von dieser wachsenden Bedeutung der auch als "International sourcing" bezeichneten Tendenz sind nahezu alle Industrie- und Dienstleistungssektoren, besonders ausgeprägt finden sich diese Strukturen jedoch in der Automobilindustrie mit inren internationalisierten Zulieferpyramiden und der Komponentenfertigung der IT-Industrie (OECD 2008: $16 \mathrm{ff}$.).

\section{Theoretische Konzepte}

Die neuere sozialwissenschaftliche Forschung über internationale Unternehmen legt eine Reihe theoretischer Konzepte vor, mit deren Hilfe die unterschiedlichen 
Verlaufsmuster, Widersprüche und Grenzen internationaler Unternehmen erklärt werden sollen. Gemeinsam ist diesen Ansätzen eine Kritik an organisationstheoretischen Konzepten ökonomischer Provenienz. Diese Konzepte fassen Unternehmen und ihr Management als rational handelnde Akteure, die den Anforderungen der Absatzmärkte durch entsprechende und optimale Strategien und Strukturen nachkommen. Ein prominenter Vertreter dieser Forschungsrichtung ist John H. Dunning (z.B. 1980), der mit seinem sog. Eklektizistischen Ansatz vor allem die managementorientierte MNU-Forschung sehr stark beeinflusst hat. Die zentralen soziologischen Argumente gegen die Auffassung eines rational begründeten "onebest-ways" von Unternehmensstrukturen sind bekannt (z.B. Hirsch-Kreinsen 2008: 115ff.): Zum einen die vielfach hohe Ungewissheit, mit denen insbesondere strategische Managemententscheidungen behaftet sind; zum zweiten das häufige Fehlen eindeutig strukturierter Entscheidungssituationen gepaart mit ihrer nur schwer überschaubaren Komplexität, zum dritten der Umstand, dass Managemententscheidungen oft zu widersprüchlichen Ergebnissen führen können, da sie zwischen verschiedenen, mehr oder weniger ungünstigen Alternativen wählen müssen, zum vierten das Problem der nicht intendierten Handlungsfolgen von Entscheidungen, die die damit verfolgten Absichten erheblich konterkarieren können und schließlich der oft übersehene Einfluss von im weitesten Sinn gesellschaftlichstrukturellen Bedingungen auf die Entscheidungsprozesse des Managements. Zugespitzt und vereinfacht lassen sich diese Argumente in mikropolitisch orientierten und institutionentheoretischen Ansätze bündeln, die seit längerem die sozialwissenschaftliche Forschung über multinationale Unternehmen stark beeinflussen. Beide Ansätze sollen im Folgenden skizziert werden.

\subsection{Mikropolitische Ansätze}

Ausgangspunkt mikropolitischer Analysen multinationaler Unternehmen ist der beschriebene empirische Befund, dass je nach konkreter Struktur, Differenziertheit und geographischer Ausdehnung eines Unternehmens eine ganze Reihe von Koordinations- und Managementproblemen auftreten können. In Anschluss an die organisationssoziologische Debatte werden unter dem Stichwort Mikropolitik Managemententscheidungsprozesse und Organisationswandel als Verhandlungs- und 
Politikprozess konzipiert. Ein Unternehmen wird hierbei als soziales System begriffen, in dem Akteure und Akteurskonstellationen mit unterschiedlichen Ressourcen, Zielen und Interessen "Machtspiele" austragen und dabei die jeweiligen Organisationsstrukturen aushandeln (Müller-J entsch 2003: 57). Anders formuliert, Unternehmensstrategien und Managemententscheidungen werden danach keineswegs allein von ökonomisch-rationalen Prämissen und Kriterien bestimmt, sondern zugleich von den in Unternehmen gegebenen Macht- und Interessenkonstellationen und den eingespielten Mustern der Abstimmung und Konfliktlösung. Bezogen auf internationale Unternehmen wird dieser Zusammenhang insbesondere in Hinblick auf das Verhältnis von Mutter- und Tochtergesellschaften diskutiert (z.B. Morgan 2005; Dörrenbächer 2006). Ausgegangen wird von einem Modell eines internationalen Unternehmens, das nicht hierarchisch von einer Zentrale aus gesteuert wird, sondern das einen polyzentrischen Charakter aufweist. Hedlunds Konzept eines "heterarchischen Unternehmens" folgend (Hedlund 1986) wird davon ausgegangen, dass die Tochtergesellschaften internationaler Unternehmen gegenüber einer Konzernzentrale strategisch autonome Einheiten seien. Sie leisten nicht nur einen eigenständigen Beitrag zur Strategie eines Gesamtunternehmens, sondern verfolgen dabei durchaus auch eigene Interessen, die eine solche Gesamtstrategie beeinflussen, ja konterkarieren können. Voraussetzungen hierfür sind spezifische Fähigkeiten und Kompetenzen von Tochtergesellschaften, die sich sowohl in der Durchsetzungsfähigkeit und Position des jeweiligen lokalen Managements als auch in den jeweiligen Standortbedingungen und den damit verfügbaren Ressourcen begründen können. Die Literatur verweist auf eine ganze Reihe sehr verschiedener konkreter Konflikte innerhalb eines MNU, die aus dieser Situation resultieren können (129 f.): Zum einen steht das Kontrollinteresse einer Unternehmenszentrale stets in latenten Widerspruch zu dem Autonomieinteresse einer Tochtergesellschaft. Zum zweiten geht es um die Frage der Verteilung von Erträgen wie auch Verlusten. Zum dritten können Fragen von Strategie, Organisationswandel und Investitionen zum Gegenstand von Auseinandersetzungen werden. Zum vierten wird auf das Problem Status- und Rollenkonflikte innerhalb des Managements wie aber auch auf arbeitspolitische Konflikte generell zwischen verschiedenen Unternehmensstandorten verwiesen. Zum fünften wird auf eine ganze Reihe sehr unterschiedlicher 
Koordinationsmechanismen multinationaler Unternehmen verwiesen, die sowohl formell-offiziellen als auch informellen Charakter haben können.

In einer Reihe von Arbeiten wird nun betont, dass die aus diesen Konfliktfeldern resultierenden innerorganisationalen Spannungen vor allem durch sozialintegrative Koordinationsmechanismen bewältigt werden können (z.B. Geppert/Mayer 2006; Kotthoff 2006). Es handelt sich dabei um Momente, die häufig auch unter dem Begriff der „Unternehmenskultur“ zusammengefasst werden. Kern dieser Mechanismen sind beispielsweise Kommunikations- und Abstimmungsprozesse, unternehmensweit aufgelegte Rationalisierungsprogramme sowie gewandelte Prinzipien der Personalpolitik. Das Management auf seinen verschiedenen Ebenen ist zugleich Träger und Adressat dieser Mechanismen. Über sie sollen nicht nur die Abstimmung und Vereinheitlichung von Zielen und Orientierungen der Akteure im multinationalen Unternehmen erreicht, sondern auch ihre Loyalität gegenüber dem Gesamtunternehmen sichergestellt werden.

Zweifellos prägen sich die mikropolitischen Konfliktbereiche je nach der Strategievariante eines multinationalen Unternehmens unterschiedlich aus. Zugleich aber ist davon auszugehen, dass die mikropolitische Perspektive grundlegende Probleme der Koordination und des Managements multinationaler Unternehmen bezeichnet. Daher kann angenommen werden, dass die skizzierten Konfliktfelder die zentrale Voraussetzung der Strategiefähigkeit internationaler Unternehmen, nämlich die Fähigkeit zur effizienten Beherrschung des "seamless web“ zwischen den verschiedenen ausdifferenzierten Unternehmenseinheiten beschränken. Je nach Wirtschaftssektor, Prozessstruktur und Unternehmensgröße verschieden stößt danach ab einem bestimmten Punkt eine ständig weitere internationale Ausdehnung der Unternehmen an ihre Grenzen. Nicht auszuschließen ist die Gefahr einer Globalisierungsfalle, in die Unternehmen zu laufen drohen: Einerseits übersteigt der Koordinationsaufwand bei weitem die angestrebten Absatz- und Kostenvorteile, so dass die fortschreitende Internationalisierung eines Unternehmens seinen ökonomischen Sinn verliert. Andererseits befinden sich die Unternehmen dann in einer Situation, die es auf Grund der faktisch herrschenden Komplexität organisatorisch sehr schwer macht umzusteuern. 


\subsection{Institutionelle Einbettung}

Freilich macht die Debatte zugleich deutlich, dass die Entwicklung von Strukturen und Strategien multinationaler Unternehmen keineswegs hinreichend in der mikropolitischen Perspektive erklärt werden kann. Vielmehr wird auf den zugleich unübergehbaren Einfluss gesellschaftlich-institutioneller auf die Unternehmensprozesse verwiesen. Diesen Zusammenhang thematisieren institutionentheoretische Ansätze, denen zu Folge die strategischen Fähigkeiten und Strukturen von Unternehmen mit den von den jeweiligen gesellschaftlich institutionellen Bedingungen gegebenen Ressourcen in Zusammenhang zu sehen sind (z.B. Boyer/ Hollingsworth 1997; Whitley 1999; Hall/Soskice 2001). Als Ressourcen werden beispielsweise die Finanzierungsbedingungen und das je spezifische System der Corporate Governance, der politische und regulative Rahmen, die vorherrschenden Konventionen und Normen und die auf Grund eines je spezifischen Bildungs- und Berufsbildungssystems verfügbaren Kompetenzen und Qualifikationen angesehen. Diese institutionellen Arrangements entwickeln sich nationalspezifisch unterschiedlich und die Unternehmen stehen in enger Wechselwirkung mit innen. Unternehmen repräsentieren daher in dieser Sichtweise distinkte, ja divergente nationale Wirtschaftssysteme (Morgan 2005: 566). Für die Analyse multinationaler Unternehmen werden aus diesem Ansatz verschiedene Konsequenzen gezogen:

Erstens wird ein dominanter Einfluss der Bedingungen des Heimatlandes - als Heimatlandeffekte (country-of-origin effect) bezeichnet - auf die Strategien und Strukturen multinationale Unternehmen angenommen; zugespitzt findet sich diese Sicht im Titel eines einflussreichen Aufsatzes „Global or Stateless Firms are National Corporations with International Operations" (Hu 1992). Ähnlich argumentieren andere Autoren wie Ruigrok/van Tulder (1995), die davon ausgehen, dass multinationale Unternehmen auf Grund ihrer sunk costs und eingespielten Praktiken, im Heimatland erprobte Organisations- und Strategiekonzepte mehr oder weniger direkt auf ihre Standorte im Ausland übertragen. So werden als Beispiel für u. U. weitreichende Heimatlandeffekte in der Literatur US-amerikanische Industrieunternehmen angeführt (Tempel et al. 2005: 187f.). Ihnen sei in der Vergangenheit 
nach dem 2. Weltkrieg auf Grund der weit verbreiteten Vorstellung, dass amerikanische Praktiken einen „one-best-way“ der Rationalisierung darstellen, der Transfer ihrer Heimatlandpraktiken und Strukturen an ausländische Standorte relativ problemlos gelungen. Ähnliches lässt sich seit Beginn der 1990er Jahre bei der Internationalisierung deutscher Unternehmen in Richtung mittel- und osteuropäischer Transformationsländer beobachten. Der Umbruch und die strukturelle Offenheit dieser Länder ermöglichten es den westlichen Unternehmen, erfolgreiche Fabrik- und Organisationsmodelle an die neuen Standorte relativ direkt zu transferieren (Bluhm 2007).

Zweitens werden in der institutionalistischen Perspektive insbesondere Gastlandeffekte auf multinationale Unternehmen betont (host-country effect). Kern dieses Gedankens ist, dass die Übertragung und Implementation eingespielter Praktiken und Strukturen stets an die institutionellen Gegebenheiten des Gastlandes, in dem sich der jeweilige Auslandsstandort befindet, angepasst werden müssen (z.B. Tempel et al. 2006). Dabei werden multinationale Unternehmen aber nicht als auf unterschiedliche Bedingungen passiv reagierende Organisationen angesehen, die sich lediglich an die spezifischen Bedingungen des Gastlandes anpassen. Vielmehr entwickeln danach Unternehmen Strategien, um die im Heimatland eingespielten Praktiken und Strukturen mit den jeweiligen Bedingungen des Gastlandes abzugleichen. Insofern werden für die Unternehmen Handlungsspielräume unterstellt, die einerseits durch die institutionellen Gegebenheiten des Gastlandes begrenzt, andererseits durch diese eröffnet werden. Konkret zeigen dies verschiedene Studien multinationaler Unternehmen in Hinblick auf den Einfluss der je nationalspezifisch verschiedenen Systeme der industriellen Beziehungen: So eröffnet umgekehrt das US-System für deutsche Unternehmen die Möglichkeit, antigewerkschaftliche Praktiken an ihren US-Standorten einzuführen, die den deutschen Vertretungstraditionen der Sozialpartnerschaft deutlich zuwider laufen. Umgekehrt sind US-Unternehmen vor dem Hintergrund der relativ offenen Strukturen ihres Heimatlandes in Deutschland mit einem für sie vergleichsweise eng geregelten und daher schwer übergehbaren System der Interessenvertretung konfrontiert. Dieses hindert sie, ihre heimatliche antigewerkschaftliche Politik auf das Gastland zu übertragen. Die häufige Unübergehbarkeit der Institutionen eines Gastlandes für 
multinationale Unternehmen zeigt eindringlich auch der Fall des Scheiterns des amerikanischen Einzelhandelskonzerns Wal-Mart in Deutschland zu Beginn der 2000er Jahre. Als Grund hierfür gilt, dass das Unternehmen versuchte, seine in den USA überaus erfolgreiche Verkaufsstrategie völlig unmodifiziert in seinen deutschen Filialen zu realisieren. Ignoriert wurden damit die extreme Konkurrenzsituation des deutschen Einzelhandelsmarktes, seine spezifischen vermachteten Zulieferstrukturen und vor allem die vorherrschenden Konsumgewohnheiten großer Kundengruppen, die konträr zu US-amerikanischen stehen. Die Konsequenz war ein ökonomisches "Fiasko“, das auch durch spätere Anpassungsmaßnahmen nicht mehr verhindert werden konnte (Knorr/Arndt 2003).

Genau genommen aber können beide Sichtweisen als zu wenig differenziert angesehen werden. Denn je nachdem welche Aspekte der Internationalisierung von Unternehmen untersucht werden, lassen sich unterschiedliche Einflüsse, Heimat-, Gast- und Drittlandeffekte nachweisen (Dörrenbächer 2006: 128). Anders formuliert, diese einzelnen Einflüsse setzen sich in den seltensten Fällen geradlinig und reibungslos durch. Üblicherweise muss vielmehr von konfliktgeladenen und widersprüchlichen Verlaufsformen ausgegangen werden. Betont wird daher, dass die Strukturen und Strategien multinationaler Unternehmen in der Regel einen "hybriden“ Charakter aufweisen (Morgan 2005: 567ff.). Obgleich die Unternehmenszentrale und die Praktiken des Heimatlandes sehr einflussreich seien, müssen sie stets an die spezifischen Kontexte der Auslandsstandorte angepasst werden. Ergebnis sei ein Unternehmensmodell, das weder allein Heimatlandeffekte noch ausschließlich Gastlandeffekte widerspiegelt. Vielmehr sei es von einem dyadischen Zusammenspiel zwischen den unterschiedlichen Heimat- und Gastlandeinflüssen gekennzeichnet.

\subsection{Transnationales soziales System}

Generalisiert man diese institutionentheoretische Perspektive, so kann davon gesprochen werden, dass Zusammenspiel zwischen Heimat- und Gastlandeinflüssen auf die Strukturen und Strategien multinationaler Unternehmen führt zu einer sozialen Ebene spezifischer institutioneller Settings und Akteurskonstellation (Morgan 
2005: 569). Es verschiebt sich dabei nicht nur die bisherige I dentität von Raum, Zeit und sozialen Prozessen, sondern es entstehen neue raum-zeitliche und soziale Konstellationen. Anders formuliert, es entstehen neue soziale Situationen, für die ein neues Mischungsverhältnis von Raum, Zeit und sozialen Prozessen charakteristisch ist (Amin 2002). Instruktives Beispiel hierfür ist die beschriebene transnationale Unternehmensstrategie: Die Netzwerkstrukturen eines solchen Unternehmens können bildlich auch als Korridore angesehen werden, innerhalb denen nach unternehmensspezifischen Regeln grenzüberschreitend die verschiedenen Unternehmensstandorte mit ihren je spezifischen lokalen Kontexten, in die sie eingebunden sind, miteinander verbunden sind und koordiniert werden. Dies erfordert zum einen die Festlegung von Schnittstellen zwischen den verschiedenen Standorten, zum zweiten die Entwicklung bestimmter Regeln der laufenden Kooperation, Kommunikation, Entscheidung etc. und schließlich erfordert dies in gewisser Hinsicht die wechselseitige Anpassung der verschiedenen Standorte an das übergreifende Regelsystem. Damit entsteht ein neues grenzüberschreitendes Unternehmen mit einer inm eigenen Struktur und Handlungslogik, die sich konkret etwa festmachen lassen an der Tendenz zur unternehmensspezifischen Vereinheitlichung der Arbeitsorganisation, einer unternehmensweit abgestimmten Personalpolitik und einer unternehmensübergreifenden Innovationsstrategie.

In generalisierender Perspektive spricht die sozialwissenschaftliche Globalisierungsdebatte daher von der Herausbildung transnationaler Sozialräume: Bezeichnet wird damit ein Prozess der Entstehung von Zugehörigkeitsgefühlen, kultureller Gemeinsamkeiten, Kommunikationsverflechtungen, Arbeitszusammenhängen und darauf bezogener sozialer Regulierungen, die nationalstaatliche Grenzen transzendieren (Pries 2008: 264). Verstanden wird darunter die Ausweitung und Vertiefung pluri-lokaler und grenzüberschreitender - ökonomischer, politischrechtlicher, sozial-kultureller etc. - Austauschbeziehungen, die neue soziale Räume jenseits der gewohnten Ortsgebundenheiten von Ankunfts- und Zielregion entstehen lässt. Es geht um Systemzusammenhänge, die grenzüberschreitenden Charakter haben, die sich auf bestimmte soziale und ökonomische Bereiche und Funktionszusammenhänge beziehen und die ein Mindestmaß an Dauerhaftigkeit und 
Stabilität aufweisen. Ein solches transnationales soziales System lässt sich wie folgt präzisieren (Abb. 2):

- Es umfasst eine Konstellation von grenzüberschreitend handelnden Akteuren, die dauerhaft nach bestimmten Mustern interagieren, kommunizieren etc.; dies kann beispielsweise das Management eines internationalen Großunternehmens sein, das nach bestimmten Entscheidungsregeln und -kriterien handelt und das spezifischen geographisch hoch mobilen Karriere- und Aufstiegsmustern folgt, die als besonderes Merkmal des jeweiligen Unternehmens anzusehen sind und die sich deutlich von denen unterscheiden, die traditionell in einzelnen Unternehmenseinheiten in den verschiedenen Ländern praktiziert worden sind.

- Über die Interaktionen bzw. Austauschprozesse zwischen den verschiedenen transnationalen Akteuren bzw. ihren Untereinheiten etabliert sich dauerhaft ein koordinierter Fluss von Waren, Wissen und Kapital; typisches Beispiel hierfür sind die länderübergreifenden Handelsströme innerhalb internationaler Großunternehmen, die einen zunehmenden Anteil am Welthandel insgesamt ausmachen.

- Diese Austauschbeziehungen sind eingebettet in Arrangements relativ dauerhafter Regeln und Normen, z.B. unternehmensübergreifende Controlling- und Steuerungssysteme, Planungs- und Logistiksysteme, einheitliche organisatorische Regelungen und die damit verbundene unternehmensübergreifende Ideologie bzw. Kultur.

- Voraussetzung transnationaler Systemstrukturen ist eine spezifische materiale Infrastruktur, beispielsweise IuK-Technologien, Transport- und Logistiksysteme, die den Fluss von Waren, Wissen und Kapital erst ermöglichen und die Unternehmensstandorte in verschiedenen Ländern „systemisch“ zu einem transnationalen System der Produktion miteinander verknüpfen.

Transnationale Systeme stehen in enger Wechselwirkung mit den jeweiligen gesellschaftlich-institutionellen Bedingungen ihrer einzelnen Standorte. Diese beeinflussen einerseits die transnationalen Systemzusammenhänge, andererseits wirken die transnationalen Prozesse auf diese zurück. Darüber hinaus stehen sie mit zwischenstaatlichen und internationale Regelungen und Beziehungen in Zusammenhang, die oftmals transnationale Systeme erst ermöglichen. 
Das Konzept der Transnationalisierung erfasst allerdings keineswegs alle denkbaren Verlaufsmuster sozialer, politischer und ökonomischer Prozesse der Globalisierung, vielmehr bezeichnet es einen spezifischen, vermutlich zunehmend wichtigeren Fall. Davon zu unterscheiden sind einerseits die Prozesse einer weitreichenden Globalisierung wie bei den Finanz- und Kapitalbeziehungen und die der SupraNationalisierung, das heißt das Aufkommen neuer politischer Souveränitätsbereiche wie etwa die EU. Andererseits unterscheidet er sich von Tendenzen hin zu einer Aufwertung von Regionen und Wirtschaftsclustern.

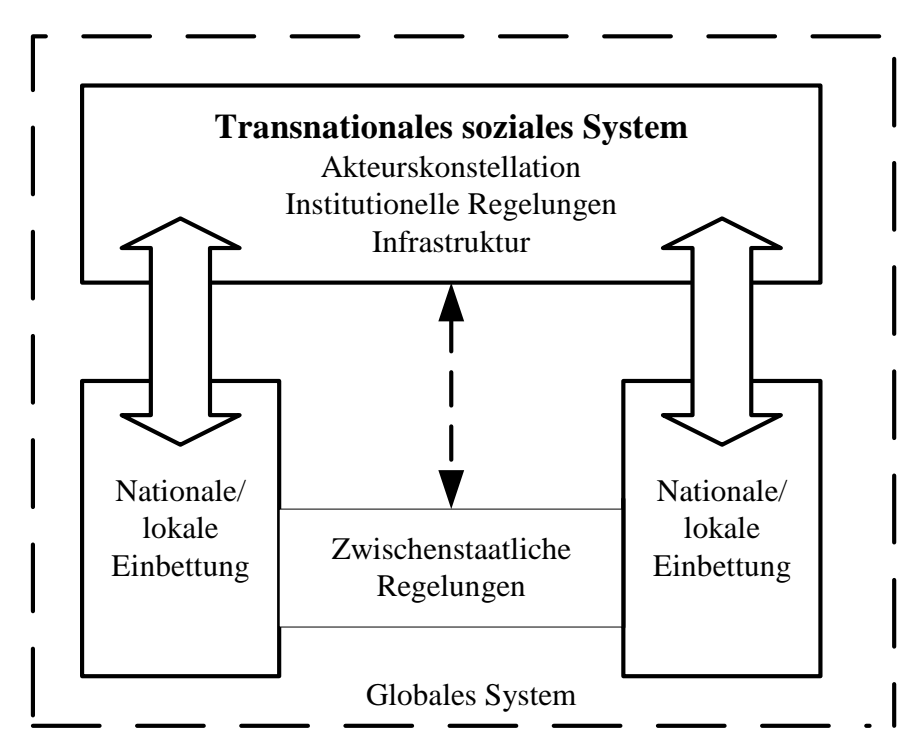

Abb. 2: Transnationales System

\section{Entwicklungsperspektiven}

Insgesamt machen die skizzierten Forschungsergebnisse folgendes deutlich: Erstens kann keineswegs von einer Konvergenz der Strukturen und Strategien internationaler Unternehmen gesprochen werden, obgleich sich Managementleitbilder, Organisationskonzepte und verfügbare Technologien im Zuge der fortschreitenden Weltmarktintegration annähern. Zu groß sind die Unterschiede der jeweiligen Heimatstandorte, der damit verbundenen Traditionen und "sunk costs“ der Unternehmen sowie die spezifischen Anforderungen verschiedener Branchen und Märkte. Zweitens ist die, vor allem in der öffentlichen Debatte genährte Vorstellung von "footloose enterprises", die problemlos und schnell ihre Standorte nach 
ökonomischem Optimierungskalkül über den Globus verteilen, bestenfalls für ein sehr kleines Segment von Unternehmen mit hoch standardisierten Produkten und Prozessen zutreffend. Für die Mehrheit internationaler Unternehmen gilt jene Situation, die mit Michael Porter (1998) als das "Paradoxon der Globalisierung" bezeichnet werden kann; dass nämlich die Bedeutung regionaler Bindungen für internationale Unternehmen eher zu-, denn abnimmt. Drittens führt die große Bedeutung heterogener Standortbedingungen für multinationale Unternehmen zu einer offenbar fortschreitenden Ausdifferenzierung und Outsourcing von Unternehmenseinheiten und einer wachsenden Bedeutung international fragmentierter Wertschöpfungsketten. Viertens wird freilich auch deutlich, dass die Internationalisierung von Unternehmen gebremst verläuft. Die Reichweite multinationaler Unternehmen umfasst nur in Ausnahmefällen den gesamten Globus. Zudem ist hierbei der Unterschied zwischen großen "global companies" und kleinen und mittleren Unternehmen (KMU) zu berücksichtigen: Auf Grund ihrer in der Regel knappen Ressourcen können letztere kaum große internationale "Sprünge“ unternehmen. Anders formuliert, ökonomische Globalisierung verläuft nicht bruchlos und unidirektional, sondern widersprüchlich und in Grenzen (Altvater/Mahnkopf 1997).

Es liegt daher die abschließende Frage nahe, welche Konsequenzen die fortschreitende ökonomische Globalisierung und die wachsende Bedeutung multinationaler Unternehmen für die Wirtschaftsstrukturen alt industrialisierter Länder wie Deutschland hat. Kann, so eine der Kernfragen der Globalisierungsdebatte (Held et al. 2005), von einer Konvergenz oder Divergenz der wirtschaftsstrukturellen Gegebenheiten verschiedener Länder gesprochen werden? In der skizzierten institutionentheoretischen Sichtweise, in der Unternehmen als einflussreiche Akteure sozialer und ökonomischer Veränderungen begriffen werden, werden hier die folgenden Antworten gegeben: Erstens wird darauf verwiesen, dass nationale Wirtschaftssysteme in den letzten J ahrzehnten zwar einem unübersehbaren Wandlungsprozess unterliegen, jedoch dieser Prozess auf Grund des hohen Beharrungsvermögens der Systeme sehr stark pfadabhängig verläuft (Boyer/ Hollingsworth 1997: 462f.). Zwar müsse von einem international wirksamen Druck auf die Angleichung bestimmter ökonomischer Handlungsparameter wie Kosten und 
Löhne ausgegangen werden, jedoch legen unterschiedliche Institutionensysteme wie die industriellen Beziehungen, Ausbildungssysteme und Arbeitsmärkte, verschiedene politisch-administrative Regelungen wie insbesondere auch unterschiedliche gesellschaftliche Normen und Wertsysteme je spezifische Antworten und Lösungen auf die im Prinzip gleichen Probleme und Herausforderungen in der globalisierten Ökonomie nahe. Oftmals stabilisieren gerade auch große multinationale Unternehmen über ihre stabilen Standortbindungen die je gegebenen institutionellen Regelungen.

Zweitens wird diese Sichtweise pfadabhängiger Entwicklungsverläufe allerdings differenziert. Denn unabweisbar sind der weltweite Druck auf Kostensenkung und Produktivitätssteigerung und die Konkurrenz ganzer Länder und Regionen um das international floatierende Kapital und die Investitionen insbesondere der multinationalen Großunternehmen. Angenommen wird daher ein Kostenwettlauf zwischen Ländern und Regionen und ein dadurch induzierter Erosionsprozess gewachsener Wirtschaftsstrukturen in Richtung eines in weiten Bereichen sinkenden Kosten- und Wohlstandsniveaus und steigender sozialer Ungleichheiten (Hirsch 1995). Als typisches Beispiel hierfür gelten arbeitsintensive Industriesektoren, die in nahezu allen westlichen Ländern einem massiven Konkurrenzdruck aus Ländern mit niedrigerem Kosten- und Lohnniveau ausgesetzt sind und die mit Strategien der Personalkostenminimierung und der Verlagerung von Produktionsstandorten in Länder mit niedrigen Kostenniveaus versuchen, diesem Druck auszuweichen.

Drittens wird in der Literatur ein Szenario skizziert, dass als fortschreitende „sektorale Spezialisierung“ gefasst werden kann (Streeck 2004). Ausgegangen wird dabei von einer Tendenz der Nutzung und des Ausbaus von nationalen und regionalen Spezialisierungsvorteilen mit der Konsequenz, dass sich Divergenzen entlang der bisher verfolgten wirtschaftsstrukturellen Entwicklungspfade verstärken. Angesprochen wird damit jener oben angedeutete Prozess, der die wachsende Bedeutung regionaler Agglomerationen im Kontext der Globalisierung begründet und multinationalen Unternehmen komparative Wettbewerbsvorteile auf Grund einer vertieften regionalen Spezialisierung verschafft (Porter 1998). Vorangetrieben wird er in dieser Sicht daher von multinationalen Unternehmen, die sich dadurch Konkurrenzvorteile verschaffen wollen wie aber auch von politischen Stellen, die 
diesen Prozess fördern. Erreicht wird dies durch wirtschafts-, technologie-, sozialund bildungspolitische Maßnahmen, die auf den Ausbau der Spezialisierungsvorteile ausgerichtet sind sowie entsprechend angepasste institutionelle Regelwerke, wie etwa die des Arbeitsmarktes und der industriellen Beziehungen. Insgesamt zielt dies auf die möglichst optimale Ausschöpfung nationaler und regionaler Ressourcen. Insgesamt wird davon ausgegangen, dass jene nationalen und regionalen Wirtschaftssysteme Entwicklungschancen entlang ihrer eingespielten Strukturen haben, die auf Grund ihrer Spezialisierung komparative Wettbewerbsvorteile im Kontext einer fortschreitenden Weltmarktverflechtung erwarten lassen, während andere unter den Druck einer verschärften Konkurrenz geraten und langfristig nur mehr geringe Entwicklungschancen haben. Resultat ist eine zunehmende Ungleichheit zwischen verschiedenen Regionen und Ländern. Sie unterscheiden sich je nach ihren wirtschaftlichen Kernsektoren, ihren dominanten Branchen und Technologien und ihren damit verbundenen Positionen und Entwicklungspotentialen in der internationalen Arbeitsteilung. Generalisiert man dieses Szenario, so kann weder von einer Tendenz zur Konvergenz noch zur Divergenz bestehender Wirtschaftsstrukturen ausgegangen werden. Vielmehr bestätigt sich auch in diesem Fall das bekannte Bild vom "patchwork“ sozialer Systeme, das als Merkmal der gegenwärtigen sozialen und ökonomischen Entwicklung moderner Gesellschaften anzusehen ist.

\section{Weiterführende Literatur}

Held, David/McGrew, Anthony/Goldblatt, David/Perraton, Jonathan (2005). Global Transformations. Cambridge (UK): Polity Press.

Mense-Petermann, Ursula/ Wagner, Gabriele (Hrsg.) (2006). Transnationale Konzerne. Ein neuer Organisationstyp? Wiesbaden: VS.

Morgan, Glenn (2005). Understanding Multinational Corporations. In: S. Ackroyd/ R. Batt/P. Thompson/S. Tolbert (Arsg.), The Oxford Handbook of Work \& Organization (S. 554-576). Oxford: UP.

Welge, Martin K./Holtbrügge, Dirk (2003). Internationales Management. Stuttgart: Schäffer-Poeschel.

\section{Im Text erwähnte Quellen:}

Abele, Eberhard/Kluge, Jürgen/Näher Ulrich (Hrsg.) (2006). Handbuch Globale Produktion. München: Hanser 
Altvater, Elmar/Mahnkopf, Birgit (1997). Grenzen der Globalisierung. Ökonomie, Okologie und Politik in der Weltgesellschaft. Münster: Westfälisches Dampfboot.

Amin, Ash (2002). Spatialities of Globalisation. Environment and Planning A, 34 (3) 385-399.

Bartlett, Christopher A./Ghoshal, Sumantra (1989). Managing Across Boarders. Boston: Harvard Business School Press.

Behr, Marhild von/Hirsch-Kreinsen, Hartmut (1998). Globale Produktion und Industriearbeit - Arbeitsorganisation und Kooperation in Produktionsnetzwerken. Frankfurt a.M./New York: Campus.

Bélanger, Jacques/Berggren, Cristian/Björkman, Torsten/Köhler, Christoph (Eds.) (1999): Being Local Worldwide. ABB and the Challenge of Global Management. Ihtaca, London: Cornell UP.

Beyer, Jürgen (2001). "One best way" oder Varietät? Strategischer und organisatorischer Wandel von Großunternehmen im Prozess der Internationalisierung. Köln: Max-Planck-Institut für Gesellschaftsforschung, Discussion Paper 01-2.

Beyer, Jürgen (2006). Verfestigte institutionelle Vielfalt? Köln: Max-Planck-Institut für Gesellschaftsforschung, Forschungsberichte aus dem MPIfG 2.

Bluhm, Katharina (2007). Experimentierfeld Ostmitteleuropa? Deutsche Unternehmen in Polen und der Tschechischen Republik. Wiesbaden: VS.

Boyer, Robert/Freyssenet, Michel (2003). Produktionsmodelle. Eine Typologie am Beispiel der Automobilindustrie. Berlin: Edition Sigma.

Boyer, J. Rogers/Hollingworth, Robert (Eds.) (1997). Contemporary Capitalism: The Embeddedness of Institutions. Cambridge: Cambridge University Press.

Busch, Klaus (1974). Die multinationalen Konzerne - Zur Analyse der Weltmarktbewegungen des Kapitals. Frankfurt a. M.: Suhrkamp.

Cameron, Rondo (1992). Geschichte der Weltwirtschaft, Bd. 2: Von der Industrialisierung bis zur Gegenwart. Stuttgart: Klett Cotta.

Caroll, William K./Fennema, Meindert (2002). Is There a Transnational Business Community? International Sociology, 17 (3), 393-419.

Dörrenbächer, Christoph (1999). Vom Hoflieferanten zum Global Player. Berlin: Edition Sigma.

Dörrenbächer, Christoph (Hrsg.) (2003). Modelltransfer in multinationalen Unternehmen. Strategien und Probleme grenzüberschreitender Konzernintegration. Berlin: Edition Sigma.

Dörrenbächer, Christoph (2006). Mikropolitik in Multinationalen Unternehmen: Konturen eines neuen Forschungsfeldes In: U. Mense-Petermann/G. Wagner (Hrsg.), Transnationale Konzerne. Ein neuer Organisationstyp? (S. 123-152). Wiesbaden: VS.

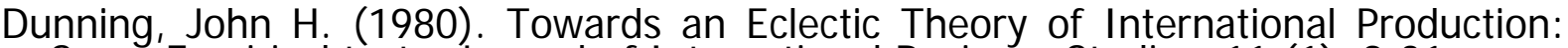
Some Empirical tests. J ournal of International Business Studies, 11 (1), 9-31.

Dunning, John $H$. (1993). Multinational Enterprises and the Global Economy. Wokingham: Addison-Wesley.

Faust, Michael/Voskamp, Ulrich/Wittke, Volker (2004). European restructuring in a Global Economy: Fragmentation and'Relocation of Value Chains. SOFI Göttingen.

Fröbel, Folker/Heinrichs, Jürgen/Kreye, Otto (1977). Die neue internationale Arbeitsteilung. Hamburg: Rowolth

Ghemawat, Pankaj (2007): Redefining Global Strategy. Boston: Harvard Business School Press

Geppert, Mike/Mayer, Michael (2006). Introduction. In: M. Geppert/M. Mayer (Hrsg.), Global, National and Local Practices in Multinational Companies (S. 1-16). Basingstoke: Palgrave.

Hall, Peter A./Soskice, David (Hrsg.) (2001). Varieties of Capitalisms. The Institutional Foundations of Comparative Advantage. Oxford: Oxford UP. 
Hartmann, Michael (1999). Auf dem Weg zur transnationalen Bourgeoisie? Leviathan, $1,112-141$.

Held, David/McGrew, Anthony/Goldblatt, David/Perraton, Jonathan (2005). Global Transformations. Cambridge (UK): Polity Press.

Hedlund, Gunnar (1986). The Hypermodern MNC - A Heterarchy? Human resource Management, 25, 9-25.

Hirsch, Joachim (1995). Der nationale Wettbewerbsstaat. Staat, Demokratie und Politik im globalen Kapitalismus Berlin, Amsterdam: Edition ID-Archiv,

Hirsch-Kreinsen, Hartmut (1998). Organisation und Koordination eines transnationalen Unternehmensnetzwerks. In: $M$. von Behr/H. Hirsch-Kreinsen (Hrsg.), Globale Produktion und Industriearbeit (S. 37-62). Frankfurt a.M., New York: Campus.

Hirsch-Kreinsen, Hartmut (2008). Wirtschafts- und Industriesoziologie. Grundlagen, Fragestellungen, Themenbereiche. 2. aktual. Aufl. Weinheim, München: J uventa.

Hirsch-Kreinsen, Hartmut/Wannöffel, Manfred (Hrsg.) (2003): Netzwerke kleiner Unternehmen. Praktiken und Besonderheiten internationaler Zusammenarbeit. Berlin: Edition Sigma.

Hu, Y.S. (1992). Global or Stateless Firms are National Corporations with International Operations. California Management Review, 34 (2), 107-126.

Kennedy, Paul (1991). Aufstieg und Fall der großen Mächte. Frankfurt a.M.: Fischer.

Kinkel, Steffen (Hrsg.) (2004). Erfolgsfaktor Standortplanung. In- und ausländische Standorte richtig bewerten. Berlin, Heidelberg, New York: Springer.

Kinkel, Steffen/Lay, Gunter (2000). Internationale Präsenz der deutschen Investitionsgüterindustrie, Mitteilungen aus der Produktionserhebung Nr. 16, März. Karlsruhe: Fraunhofer ISI.

Kinkel, Steffen/Maloca, Spomenka (2008). Produktionsverlagerungen rückläufig Ausmaß und Motive von Produktionsverlagerungen und Rückverlagerungen im deutschen Verarbeitenden Gewerbe. Mitteilungen aus der Produktionsinnovationserhebung Nr. 45; Karlsruhe: Fraunhofer ISI.

Knorr, Andreas/Arndt, Andreas (2003). Why did Wal-Mart fail in Germany? In: A. Knorr/A. Lemper/A. Sell/K. Wohlmuth (Hrsg.), Materialien. des Wissenschaftsschwerpunktes "Globalisierung der Weltwirtschaft". Bd. 24. Bremen: IWIM Universität Bremen.

Kotthoff, Hermann (2006). Wer bekommt den Kassenschlüssel hinter der Grenze? In: U. Mense-Petermann/ G. Wagner (Hrsg.), Transnationale Konzerne. Ein neuer Organisationstyp? (S. 279-299). Wiesbaden: VS Verlag.

Morgan, Glenn (2005). Understanding Multinational Corporations. In: S. Ackroyd/ R. Batt/ P. Thompson/ S. Tolbert (Hrsg.), The Oxford Handbook of Work \& Organization (S. 554-576). Oxford: UP.

Müller-J entsch, Walter (2003). Organisationssoziologie. Frankfurt a.M./New York: Campus.

Müller, Torsten/Platzer, Hans-Wolfgang/Rüb, Stefan (Hrsg.) 2006. Industrielle Beziehungen. Schwerpunktheft Globalisierung und transnationale Arbeitsbeziehungen, Jahrgang 13 (3).

OECD (2008). Staying Competitive in the Global Economy. Compendium of Studies on Global Value Chains. Paris: OECD

Ohmae, Kenichi (1990). The Borderless World: Power, Strategy in the Interlinked Economy. London: Fontana.

Porter, Michael E. (1986). Competition in Global Industries. Boston: Harvard Business School Press.

Porter, Michael E. (1998). On Competition, Boston: Harvard Business School.

Pries, Ludger (1999). Auf dem Weg zu global operierenden Konzernen? BMW, Daimler-Benz und Volkswagen: die "Drel Großen" der deutschen Automobilindustrie. München/Mering: Hampp.

Pries, Ludger (2008). Die Transnationalisierung der sozialen Welt. Frankfurt a.M.: Suhrkamp 
Ruigrok, Winfried/van Tulder, Rob (1995). The Logic of International Restructuring. London/New York: Rutledge.

Schamp, Eike (2000). Die Stabilität regionaler Unternehmenscluster - Ergebnisse wirtschaftsgeographischer Forschung. In: H. Hirsch-Kreinsen/A. Schulte (Hrsg.), Standortbindungen (S. 61-102). Berlin: edition sigma.

Schmierl, Klaus (1998). Amorphie im "Normierten Verhandlungssystem" - Wandel industrieller Beziehungen im internationalen Unternehmensverbund. In: $M$. von Behr/ H. Hirsch-Kreinsen (Hrsg.), Globale Produktion und Industriearbeit (S. 161208). Frankfurt a.M., New York: Campus.

Schulte, Anja (2002). Das Phänomen der Rückverlagerung. Internationale Standortentscheidungen kleiner und mittlerer Unternehmen. Wiesbaden: Gabler.

Scott, Allen J. (1996). Regional Motors of the Global Economy. Futures, 28 (5), 391411.

Streeck, Wolfgang (2004). Globalisierung: Mythos und Wirklichkeit. MPIfG Working Paper 04/4, September.

Sturgeon, Timothy/van Biesebroeck, Johannes/Gereffi, Gary (2008). Value Chains, networks and clusters: reframing the global automotive industry. Journal of Economic Geography, 8, 297-321.

Tempel, Anne/Wächter Hartmut/Walgenbach, Peter (2005). Multinationale Unternehmen und internationales Personalmanagement. Eine vergleichende institutionalistische Perspektive. Zeitschrift für Personalforschung, 19 (2), 181-202.

Tempel, Anne/Wächter, Hartmut/Walgenbach, Peter (2006): The Comparative Institutional Approach. In: M. Geppert/M. Mayer $\left(\mathrm{Hrsg}_{\mathrm{f}}\right)$, Global, National and Local Practices in Multinational Companies (S. 17-37). Basingstoke: Palgrave.

UNCTD (United Nations Conference on Trade and Development) (2007): World Investment Report 2007. Transnational Corporations, Extractive Industries and Development. New York, Geneva: United Nations Publications.

Wagner, Gabriele/Mense-Petermann, Ursula (2006). Zur Einleitung: Transnationale Konzerne als neuer Organisationstyp? Glokalität als Organisationsproblem. In: U. Mense-Petermann/ G. Wagner (Hrsg.), Transnationale Konzerne. Ein neuer Organisationstyp? (S. 9-32). Wiesbaden: VS.

Welge, Martin K./Holtbrügge, Dirk 2003: Internationales Management. Stuttgart: Schäffer-Poeschel.

Whitley, Richard (1999). Divergent Capitalism. The Social Structuring and Change of Business Systems. New York: Oxford University Press. 


\section{Bereits erschienene Soziologische Arbeitspapiere}

1/2003 Hartmut Hirsch-Kreinsen, David Jacobsen, Staffan Laestadius, Keith Smith

Low-Tech Industries and the Knowledge Economy: State of the Art and Research Challenges

(August 2003)

2/2004 Hartmut Hirsch-Kreinsen

"Low-Technology": Ein innovationspolitisch vergessener Sektor (Februar 2004)

3/2004 Johannes Weyer

Innovationen fördern - aber wie? Zur Rolle des Staates in der

Innovationspolitik

(März 2004)

4/2004 Konstanze Senge

Der Fall Wal-Mart: Institutionelle Grenzen ökonomischer Globalisierung (J uli 2004)

5/2004 Tabea Bromberg

New Forms of Company Co-operation and Effects on Industrial Relations (J uli 2004)

6/2004 Gerd Bender

Innovation in Low-tech - Considerations based on a few case studies in eleven European countries

(September 2004)

7/2004 Johannes Weyer

Creating Order in Hybrid Systems. Reflexions on the Interaction of Man and Smart Machines

(Oktober 2004)

8/2004 Hartmut Hirsch-Kreinsen

Koordination und Rationalität

(Oktober 2004) 
9/2005 Jörg Abel

Vom Kollektiv zum Individuum? Zum Verhältnis von Selbstvertretung und kollektiver Interessenvertretung in Neue Medien-Unternehmen

(Juli 2005)

10/2005 Johannes Weyer

Die Raumfahrtpolitik des Bundesforschungsministeriums

(Oktober 2005)

11/2005 Horst Steg

Transnationalisierung nationaler Innovationssysteme

(Dezember 2005)

12/2006 Tobias Haertel

UsersAward: Ein Beitrag zur optimalen Gestaltung von Mensch-MaschineSystemen in der Logistik

(Februar 2006)

13/2006 Doris Blutner, Stephan Cramer, Tobias Haertel

Der Mensch in der Logistik: Planer, Operateur und Problemlöser

(März 2006)

14/2006 Johannes Weyer

Die Zukunft des Autos - das Auto der Zukunft. Wird der

Computer den Menschen ersetzen?

(März 2006)

15/2006 Simone Reineke

Boundary Spanner als Promotoren des Wissensmanagementprozesses

(Juli 2006)

16/2006 Johannes Weyer

Die Kooperation menschlicher Akteure und nicht-menschlicher Agenten.

Ansatzpunkte einer Soziologie hybrider Systeme

(Juli 2006)

17/2006 Jörg Abel/Sebastian Campagna/Hartmut Hirsch-Kreinsen (Hg.)

Skalierbare Organisation

- Überlegungen zum Ausgleich von Auftragsschwankungen -

(August 2006)

18/2006 Tabea Bromberg

Engineering-Dienstleistungen in der Automobilindustrie:

Verbreitung, Kooperationsformen und arbeitspolitische Konsequenzen

(Mai 2007) 
19/2006 Hartmut Hirsch-Kreinsen

Lohnarbeit

(September 2007)

20/2008 Katrin Hahn

Der Lissabon-Prozess: Das Innovationskonzept und die

Auswirkungen auf die Politikgestaltung

(März 2008)

21/2008 Anja J. Lorenz/ Johannes Weyer (Hrsg.)

Fahrerassistenzsysteme und intelligente Verkehrssteuerung.

Soziologische Analysen hoch automatisierter Verkehrssysteme (J uni 2008)

22/2008 Hartmut Hirsch-Kreinsen

Innovationspolitik: Die Hightech-Obsession

(August 2008) 